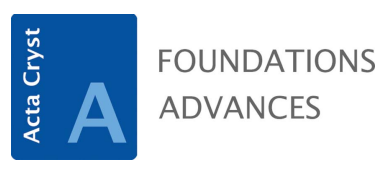

ISSN 2053-2733

Received 24 October 2019

Accepted 18 December 2019

Edited by L. D. Marks, Northwestern University, USA

Keywords: space groups; inversion symmetry; electron channelling; X-ray emission; Heusler crystals.

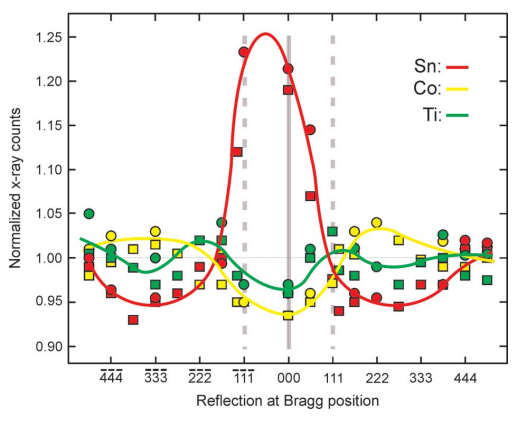

OPEN ๑ ACCESS

\section{Distinguishing space groups by electron channelling: centrosymmetric full-Heusler or non-centrosymmetric half-Heusler?}

\author{
Vidar Hansen, ${ }^{\text {a* }}$ Andrey Kosinskiy ${ }^{\mathrm{b}}$ and Johan Taftø ${ }^{\mathrm{b}}$

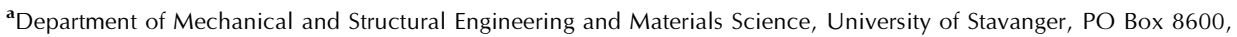 \\ Stavanger 4036, Norway, and ${ }^{\mathbf{b}}$ Department of Physics, Centre for Materials Science and Technology, University of Oslo, \\ PO Box 1048, Blindern, Oslo N-0316, Norway. *Correspondence e-mail: vidar.hansen@uis.no
}

$\mathrm{X}$-ray emission under electron-channelling conditions is used to distinguish between a non-centrosymmetric half-Heusler and a centrosymmetric fullHeusler crystal. For $\mathrm{TiCo}_{1.5+x} \mathrm{Sn}$ the space-group determination based on a Rietveld refinement procedure became challenging for increasing Co content $(x>0.2)$, while electron channelling proved successful for higher Co content $(x=0.35)$. This technique can be used on crystals as small as $(10 \mathrm{~nm})^{3}$.

\section{Introduction}

Centrosymmetric and non-centrosymmetric crystals may have the same extinctions of reflections. Potential experimental techniques to obtain structure-factor phase information, and thus to test for lack of centrosymmetry, are many-beam diffraction and standing-wave or channelling experiments with incident X-rays, neutrons or electrons. A more established technique to distinguish between centrosymmetric and noncentrosymmetric crystals is anomalous scattering of X-rays; see the pioneering work of Bijvoet et al. (1951) and, for example, Marezio (1965).

In this short communication, electron channelling is used experimentally to distinguish between a half-Heusler crystal with space group $F \overline{4} 3 m$ (No. 216) and a full-Heusler, $F m \overline{3} m$ (No. 225). The Ti-Co-Sn system was studied. The specimen fabrication, starting with arc melting, is described by Kosinskiy et al. (2016). The composition of the Heusler phase was carefully measured using an energy-dispersive X-ray spectrometer attached to a scanning electron microscope. The composition was measured to be $\mathrm{Ti}_{0.27} \mathrm{Co}_{0.49} \mathrm{Sn}_{0.24}$ with an estimated average atomic content of $\mathrm{Ti}_{4.00} \mathrm{Co}_{7.40} \mathrm{Sn}_{3.60}$ within the unit cell, based on combining lattice-parameter measurements (Kosinskiy et al., 2016) with application of Vegard's law. This unit-cell content corresponds to the chemical formula $\mathrm{Ti}_{1.00} \mathrm{Co}_{1.85} \mathrm{Sn}_{0.90}$ in the usual way of expressing the atomic content of a Heusler compound.

In this study, electron channelling is used to obtain direct information about crystal symmetry and space groups. This is a task quite different from using electron channelling to locate atoms in crystals, the latter being referred to as the ALCHEMI technique (Spence \& Taft $\varnothing, 1983$ ). Rather, it is an extension of using electron channelling to determine the polar direction of non-centrosymmetric crystals (Taftø, 1983; Jiang et al., 2002). 


\section{Experimental details}

The electron-channelling experiments were performed with $200 \mathrm{keV}$ incident electrons by operating a JEOL 2100 transmission electron microscope in the convergent-beam diffraction mode. The electron-induced element-characteristic $\mathrm{X}$-ray emission signals were detected using an EDAX energydispersive spectrometer. Measurements were done under planar channelling conditions, with reflections along the $(h h h)$ reciprocal-lattice row excited (Fig. 1). More details on electron channelling from Heusler crystals are presented in a recent paper by Hansen et al. (2018) and in another paper by Morimura \& Hasaka (2006). A focused electron beam of diameter around $10 \mathrm{~nm}$ illuminated areas of thickness around $20 \mathrm{~nm}$ near the edge of a wedge-shaped region on the specimen. The convergence of the incident beam (divergence of the Braggreflected beams, Fig. 1) corresponds to a diameter close to the length of the $g$ vector for the 111 reflection, $\left(1^{2}+1^{2}+1^{2}\right)^{1 / 2} /$ $6.06 \AA=0.29 \AA^{-1}$, where $6.06 \AA$ is the lattice parameter of the crystal. Small clean and smooth areas of specimen were prepared by crushing a sintered specimen.

\section{Discussion and conclusions}

The normalized X-ray emission from the three elements in the crystal is also shown in Fig. 1 for different incident-beam directions. These are shown for two sets of measurements. We note a significant deviation from inversion across incidence

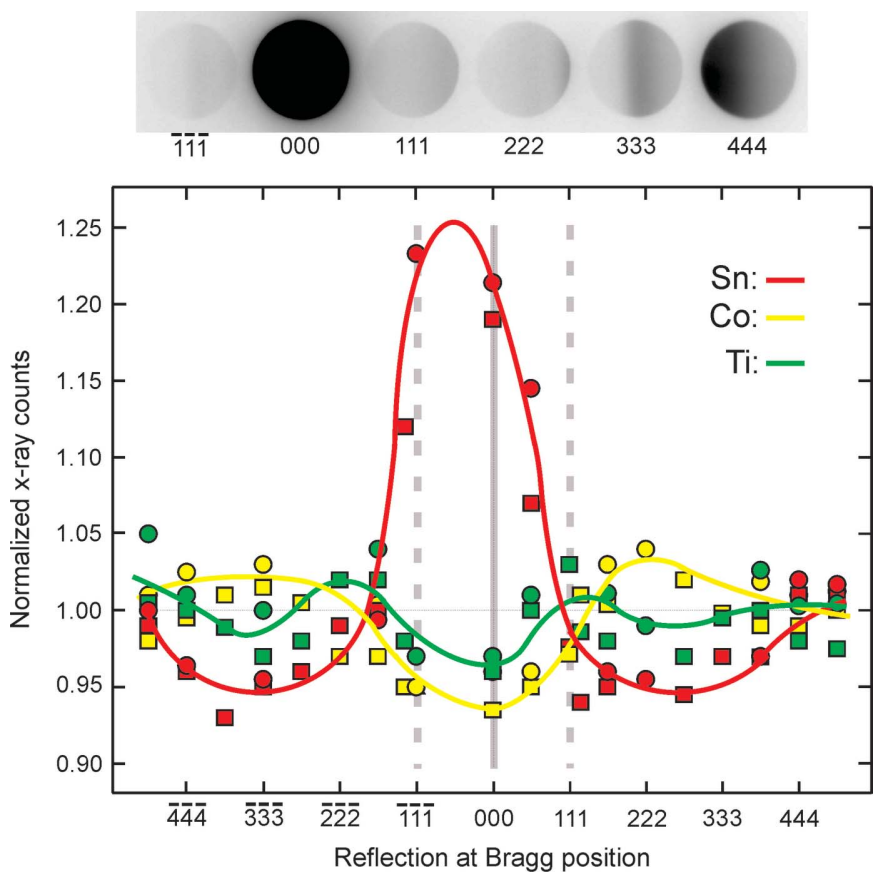

Figure 1

Normalized X-ray emission, as a function of the incident-beam direction, from the three elements of the crystal, namely the $L$ line of Sn and the $K$ lines of $\mathrm{Ti}$ and Co. The squares (red Sn, yellow Co and green Ti) and circles (red $\mathrm{Sn}$, yellow $\mathrm{Co}$ and green $\mathrm{Ti}$ ) are from two sets of measurements from different specimen regions. At the top is shown an example of the diffraction condition during the acquisition of an X-ray spectrum.
Table 1

The ratio between the X-ray emission, $\left(N_{111} / N_{\overline{111}}\right)$, from seven pairs of additional measurements with the 111 and 111 reflections at the Bragg position, respectively.

\begin{tabular}{llllllll}
\hline $\mathrm{Sn}$ & 0.81 & 0.91 & 0.91 & 0.84 & 0.89 & 0.86 & 0.83 \\
$\mathrm{Ti}$ & 1.09 & 1.07 & 1.08 & 1.08 & 1.03 & 1.08 & 1.07 \\
$\mathrm{Co}$ & 1.03 & 1.00 & 0.99 & 1.03 & 1.02 & 1.02 & 1.03 \\
\hline
\end{tabular}

parallel to the 111 planes, in particular by comparing the $\mathrm{X}$-ray yields for the $\overline{111}$ and 111 reflections at the Bragg position. Additional pairs of spectra with the $\overline{111}$ and 111 reflections at the Bragg position were acquired and the ratios are shown in Table 1. In all cases we note that the ratio for $\mathrm{Sn}$ is below unity, the ratio for $\mathrm{Ti}$ is above unity and the ratio for $\mathrm{Co}$ is close to unity.

Assuming the two-beam approximation applies, the electron wavefield intensity across the repetition unit behaves as indicated in Fig. 2 for the $\overline{111}$ and 111 reflections at the Bragg position. The amplitudes of these curves vary with thickness, as seen from the analytical expression for wavefield modulation in the two-beam approximation with absorption (diffuse scattering) (Taftø, 1983),

$$
I(x, z)=1-D \sin (2 \pi g x) \sin \left[2 \pi\left(\gamma_{1}-\gamma_{2}\right) z\right] .
$$

Here, $z$ is the distance from the entrance surface, $x$ the position within the repetition unit and $\left(\gamma_{1}-\gamma_{2}\right)$ the inverse of the period of the thickness oscillation. $D$ is a damping factor due to diffuse scattering out of the two beams (direct and Braggreflected beams), the averaging over the thickness of the crystal area which is illuminated by the incident electrons, and the convergence of the incident beam.

In the two-beam approximation the wavefield shows the same modulation, whether half-Heusler or full-Heusler.

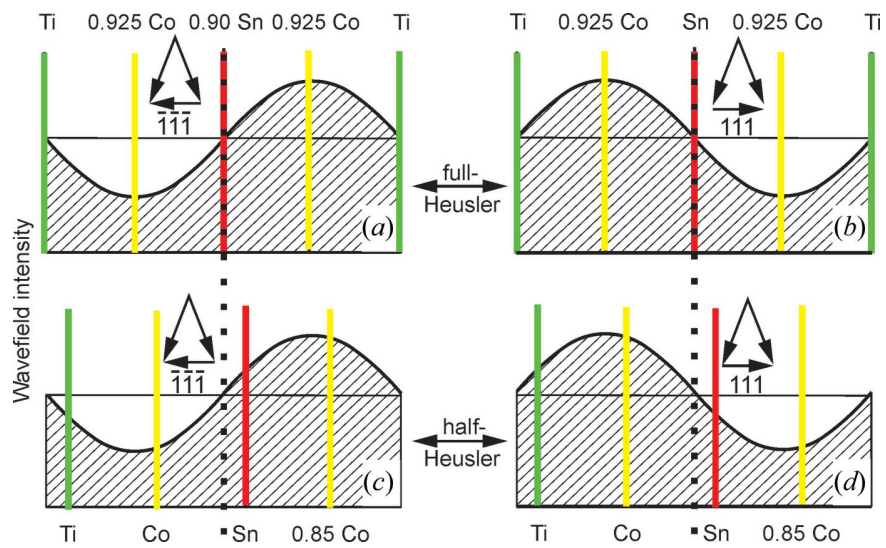

Figure 2

(a), (b) Two-beam calculations of the wavefield modulation for the centrosymmetric model, space group $F m \overline{3} m$ (No. 225), with the Bragg condition fulfilled for $(a)$ the $\overline{111}$ reflection and $(b)$ the 111 reflection. $(c)$, (d) Similarly for the non-centrosymmetric model, space group $F \overline{4} 3 m$ (No. 216). In the analytical calculations for both the centrosymmetric and noncentrosymmetric models, the origin is chosen at the same position within the figure (vertical dotted line). The origin for both models is at the position resulting in a real and positive structure factor for the 111 reflection (and thus also for the $\overline{111}$ reflection). This origin is in the Sn plane in the centrosymmetric model and the crystal had to be shifted by $25^{\circ} / 360^{\circ}$ parts on the repetition unit for the non-centrosymmetric model. 
Relative to this modulation the crystal is shifted (Fig. 2). The origin, which we choose to place in the Sn plane for the fullHeusler model, is for the half-Heusler model at the position maintaining a real and positive value of the structure factor of the 111 reflection, and thus also the $\overline{111}$ reflection.

Although not relevant for the space-group issue, we add the following comments. From the modulation calculated in Fig. 2 it is expected that the ratio $\left(N_{111} / N_{\overline{111}}\right)_{\mathrm{Ti}}$ should be the inverse of $\left(N_{111} / N_{\overline{111}}\right)_{\mathrm{Sn}}$. The average ratio for Sn is 0.87 (Table 1$)$ and the inverse is 1.15 . The observed ratio for $\mathrm{Ti}$ is only 1.07, however, which may, at least partly, be explained by the presence of around $10 \%$ of the Ti atoms on the $\mathrm{Sn}$ position.

In a recent study of the Heusler system $\mathrm{TiCo}_{1.5+x} \mathrm{Sn}$ (Kosinskiy et al., 2016), it was reported that it became exceedingly difficult from Rietveld refinement of X-ray powder diffraction data to distinguish between the noncentrosymmetric half-Heusler and the centrosymmetric fullHeusler for $x>0.2$. In the present study a clear lack of symmetry is observed for a crystal approaching the composition of a full-Heusler $(x=0.35)$. We conclude that the crystal is a non-centrosymmetric half-Heusler with space group $F \overline{4} 3 m$ (No. 216). This demonstrates that electron channelling, a technique that can be used on crystal volumes as small as $(10 \mathrm{~nm})^{3}$, is highly sensitive to lack of centrosymmetry.

\section{Acknowledgements}

We are grateful to Dr Mona W. Minde at the University of Stavanger for the composition measurements using scanning electron microscopy, and to senior engineer Ole Bjørn Karlsen at the University of Oslo for valuable discussions.

\section{Funding information}

Financial support from the Norwegian Research Council through the THELMA project (No. 2287854) is gratefully acknowledged.

\section{References}

Bijvoet, J. M., Peerdeman, A. F. \& van Bommel, A. J. (1951). Nature, 168, 271-272.

Hansen, V., Echevarria-Bonet, C., Minde, M. W. \& Taftø, J. (2018). AIP Adv. 8, 125335.

Jiang, N., Eustis, T. J., Cai, J., Ponce, F. A., Spence, J. C. H. \& Silcox, J. (2002). Appl. Phys. Lett. 80, 389-391.

Kosinskiy, A., Karlsen, O. B., Sørby, M. H. \& Prytz, Ø. (2016). Metall. Mater. Trans. E, 3, 329-336.

Marezio, M. (1965). Acta Cryst. 19, 396-400.

Morimura, T. \& Hasaka, M. (2006). Ultramicroscopy, 106, 553-560.

Spence, J. C. H. \& Taft $\varnothing$, J. (1983). J. Microsc. 130, 147-154.

Taftø, J. (1983). Phys. Rev. Lett. 51, 560-563. 